\title{
Cutaneous Lymphadenoma: A Case Report and Immunohistochemical Study
}

\author{
Taku Fujimura Aya Itoigawa Takahiro Haga \\ Setsuya Aiba
}

Department of Dermatology, Tohoku University Graduate School of Medicine, Sendai, Japan

\section{Key Words}

Cutaneous Iymphadenoma · CD1a · Foxp3 - Tumor-infiltrating lymphocytes

\begin{abstract}
Cutaneous lymphadenoma is a rare neoplasm, and characterized by a florid mononuclear cell infiltration that appears to be an integral component of the tumor. Several reports suggested that these infiltrated lymphocytes mainly consist of T cells and Langerhans cells, but no detailed phenotypical analysis of these cells has been demonstrated yet. In this report, we describe a 70-year-old Japanese patient with cutaneous lymphadenoma. In the present case, we investigated the immunohistochemical profiles of tumor-infiltrating cells in cutaneous lymphadenoma, especially focusing on the profiles of T cell subsets. In addition, there are abundant mucin deposits within the tumor stroma, and tumor lobules are composed of BerEP4-positive cells. To the best of our knowledge, this is the first report that employed immunohistochemical staining of cutaneous lymphadenoma to verify the types of cells composing such tumors with special focus on the profiles of the T cell subsets.
\end{abstract}

\section{Introduction}

Cutaneous lymphadenoma is an uncommon epithelial neoplasm with a prominent lymphoid infiltrate in the epithelial islands [1]. Cutaneous lymphadenoma was first described in 1991 by Santa Cruz et al. [2] and has been reported in the literature under various names (e.g. benign lymphoepithelial tumor of the skin and adamantinoid trichoblastoma) [3-7]. Though several reports suggest that tumor-infiltrating lymphocytes (TILs) consist of $\mathrm{CD} 1 \mathrm{a}^{+} \mathrm{S} 100^{+}$Langerhans cells and CD ${ }^{+} \mathrm{T}$ cells, no detailed phenotypical analysis of T cells has been demonstrated yet [1]. 
The histogenesis of cutaneous lymphadenoma is still debated and various hypotheses have been proposed [6]. On the basis of immunohistochemical studies, it is thought that cutaneous lymphadenoma originates from hair follicles and represents a variant of trichoblastoma [8]. However, the characteristic pattern of the mononuclear cells infiltrate is still unknown.

In this report, we employed immunohistochemical staining to verify the TILs with special focus on the profiles of $\mathrm{T}$ cell subsets and the tumor stroma.

\section{Case Report}

A 70-year-old Japanese woman visited our outpatient clinic with a 40-year history of an asymptomatic nodule on the face. On the initial visit, physical examination revealed a skin-colored, elastic-hard, well-demarcated nodule on the right cheek (fig. 1). The tumor mass was $22 \times 20 \mathrm{~mm}$ in size. We excised the tumor with a 5-mm surgical margin. Histologic examination revealed cords, lobules, and interconnected strands with a peripheral, palisaded border of basaloid-like cells and a slight admixture of central mononuclear cells (fig. $2 \mathrm{a}$ ). In addition, the tumor stroma was positive for Alucian blue (fig. 2b), and the inside of the duct was positive for AE1/AE3 (fig. 2c). CEA, CK7, CK20 were negative (data not shown). We also determined that the cells composing the tumor lobules were partially positive for BerEP4 (fig. 2d). From the above data, we diagnosed this patient as having cutaneous lymphadenoma.

Next, to evaluate the tumor-composing cells in the cutaneous lymphadenoma, we performed immunohistochemical stainings, which revealed that these mononuclear cells within the lobules and tumor stroma areas were mainly CD1 ${ }^{+}$cells ( $\underline{\text { fig. } 3 a)}$ and, CD3+, CD4+, CD7+, CD8 ${ }^{+}$and TIA-1+ $\mathrm{T}$ cells (fig. $3 \mathrm{~b}-\mathrm{e}, \mathrm{h}$ ). These tumor-infiltrating $\mathrm{T}$ cells were mainly $\mathrm{CD} 45 \mathrm{Ro}^{+}$memory $\mathrm{T}$ cells, and several $\mathrm{CD}_{4} 5 \mathrm{RA}^{+}$cells were also detected (data not shown). In addition, several Foxp3+ Tregs were detected within the lobules (fig. 4). CD20+ cells and $\mathrm{CD}^{+} 8^{+}$cells were also detected within and around the lobules (fig. $3 f, g$ ). $\mathrm{CD}_{56} 6^{+}$cells were not detected in our case (data not shown).

\section{Discussion}

We describe a case of cutaneous lymphadenoma, which is known to be infiltrated with various types of lymphocytes [1]. In this report, we employed immunohistochemical staining to verify the tumor-composing lymphocytes, especially focusing on the profiles of the T cell subsets.

Cutaneous lymphadenoma is a rare neoplasm that arises mainly on the skin of the head and neck [1-8]. The exact pathogenesis remains unknown and the histogenesis is uncertain. Clinically, the tumor presents as a slow growing, skin colored papule, nodule, or plaque without erythema or other signs of tumor-associated inflammation [5]. The histological differential diagnosis includes clear cell BCC, clear cell syringoma, trichoepithelioma, dermal thymus and malignant lymphoepithelioma-like carcinoma [8]. However, a lymphoid cell infiltrate within the tumor lobules is not prominent in such tumors.

Previously, several reports suggested that, in cutaneous lymphadenoma, cells within both the lobules and interstitial area consist of CD1 $\mathrm{a}^{+}$Langerhans cells, CD30+ $\mathrm{T}$ or $\mathrm{B}$ cells, and $\mathrm{CD}^{+} \mathrm{T}$ cells $[1,4]$. More detailed investigation has not been performed yet. In our case, immunohistochemical staining for CD3, CD4, CD8, TIA-1, CD20, CD68, Foxp3 and CD56 was found in the phenotypic analysis of the cutaneous lymphadenoma- 
infiltrating cells. We investigated the expression of CD45RA and CD45Ro on infiltrating lymphocytes, and found that, in cutaneous lymphadenoma, infiltrating cells mainly consist of memory T cells. We also detected prominent infiltration of Foxp3+ Treg cells, as we previously reported in other types of skin tumors [9]. In addition, we also focused on the tumor stroma. In our case, the tumor lobules consisted of AE1/AE3 ${ }^{+}$and BerEP4 ${ }^{+}$cells, and within the lobules, stroma is mainly positive for AE1/AE3. In contrast, on the outside of the lobules, the stroma showed abundant mucin deposits. As we previously reported the profiles of TILs in metastatic malignant melanomas [10], normally in malignant tumor microenvironment, specific lymphocytes such as Tregs, cytotoxic T lymphocytes, etc. are increasing. In the present case, there is no tendency of increasing numbers of effector $\mathrm{T}$ cells, suppressor cells, nor antigen-presenting cells. These observations may be because cutaneous lymphadenoma is not a malignant tumor but a benign neoplasm of follicular or lymphoepithelial differentiation. Our present case suggested that the pathogenesis of tumor-infiltrating cells in cutaneous lymphadenoma is different from TILs in malignant skin tumors. Though we could not further assess the functions of these infiltrating cells, our present study suggests, at least, that these varieties of lymphocytes may have played roles in tuning and maintaining the homeostasis of this tumor.

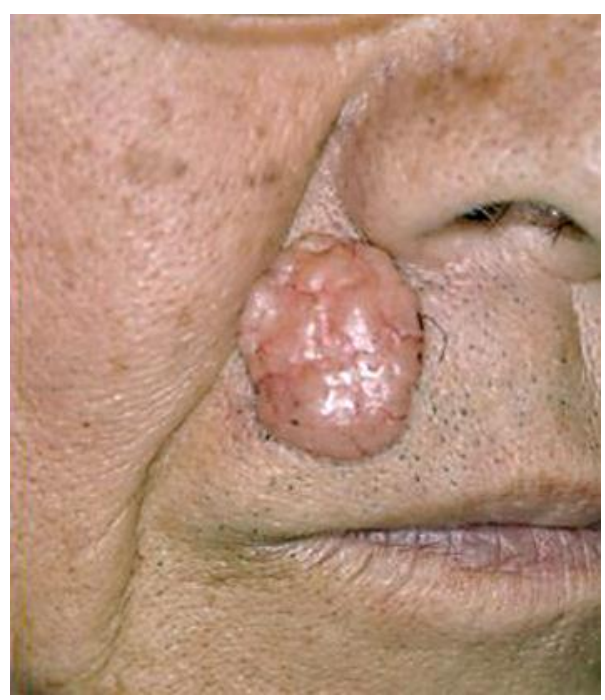

Fig. 1. Skin-colored, elastic-hard, well-demarcated nodule on the right cheek. The tumor mass was $20 \times 22 \mathrm{~mm}$ in size. 

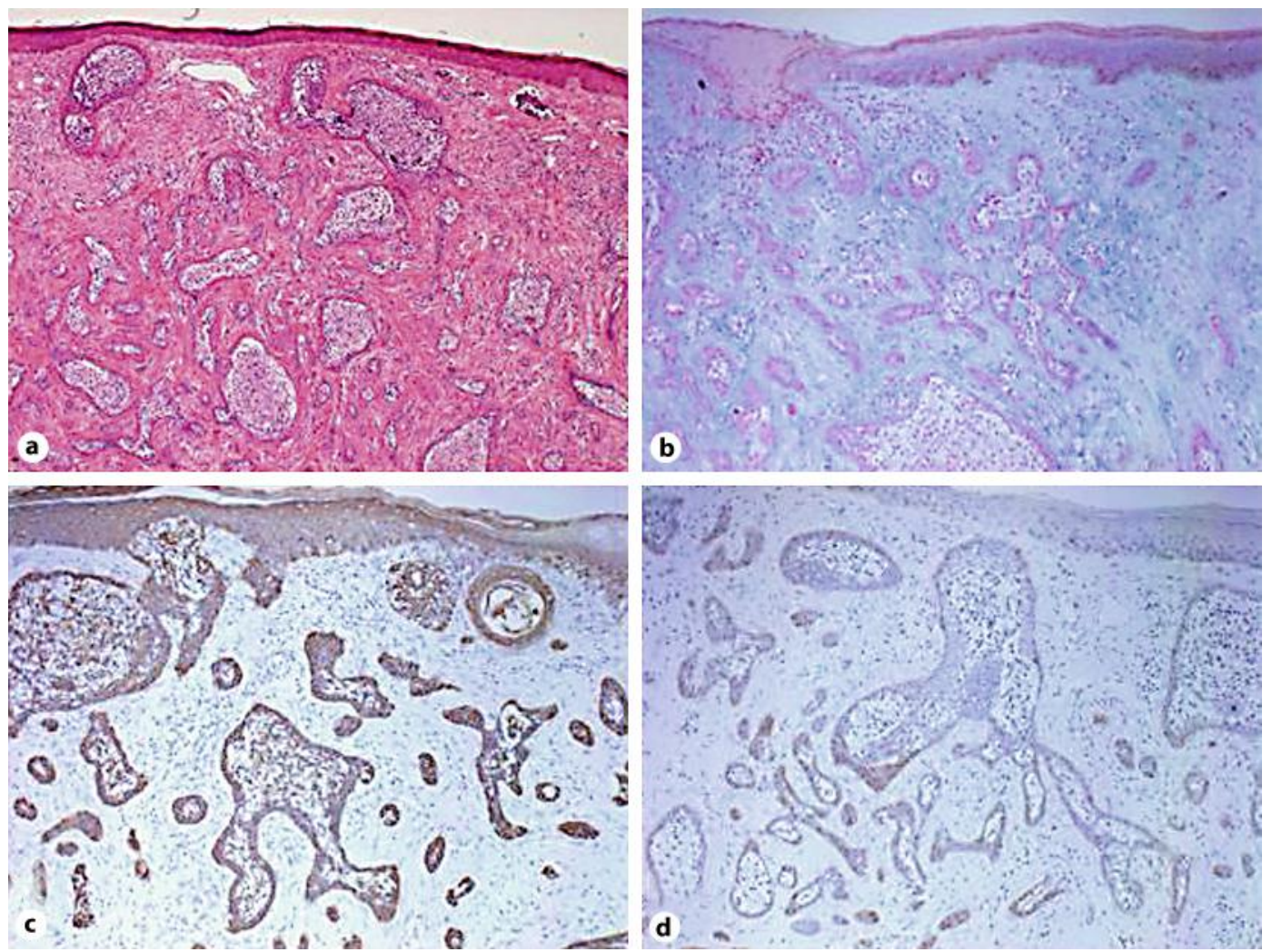

Fig. 2. Cords, lobules, and interconnected strands with peripheral, palisaded border of basaloid-like cells and a slight admixture of central mononuclear cells (original magnification $\mathbf{a} \times 50, \mathbf{b}-\mathbf{d} \times 200$ ). 

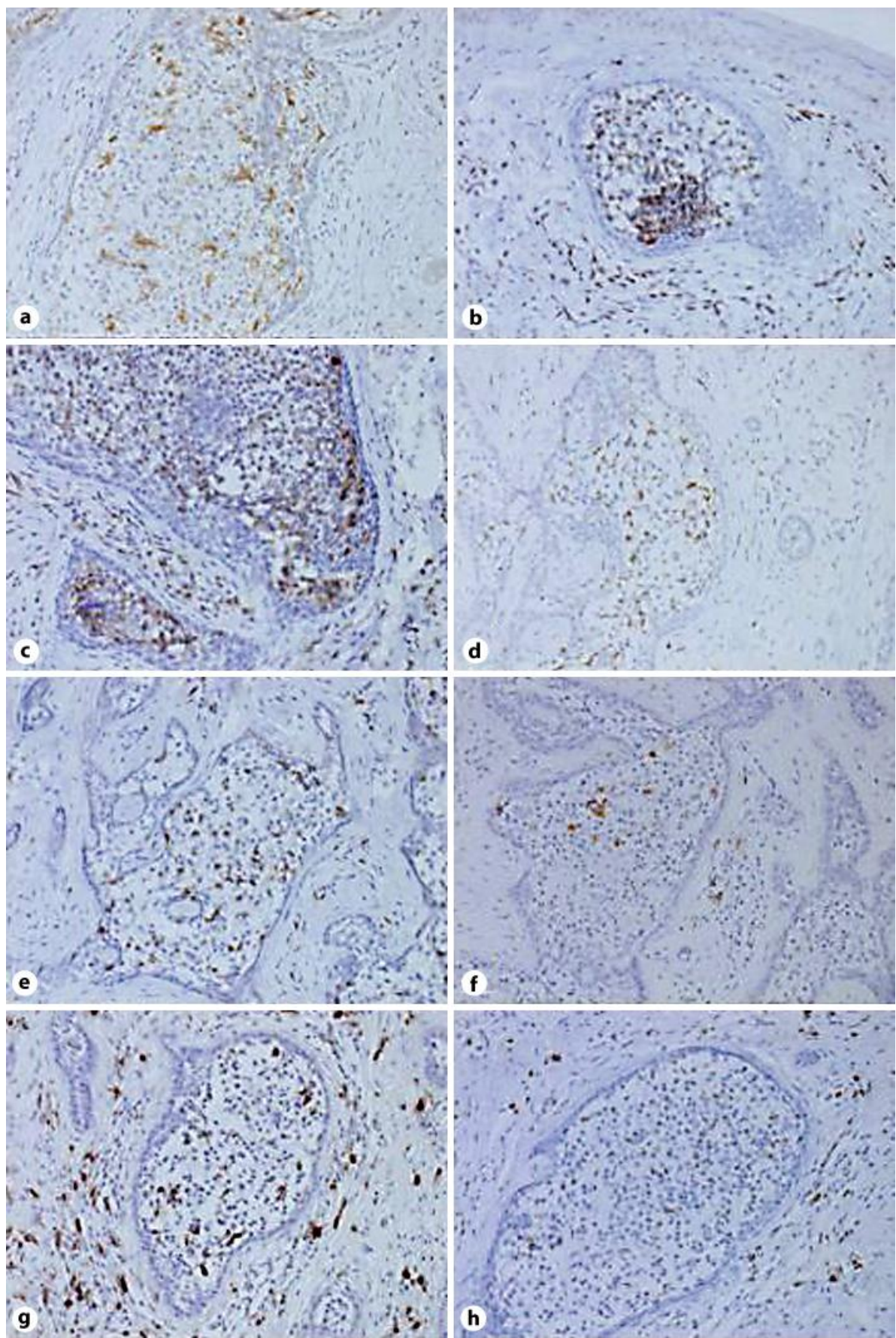

Fig. 3. Immunohistochemical stainings for cutaneous lymphadenoma. Paraffin-embedded tissue samples from the patient were stained as follows: the sections were developed with 3,3diaminobenzidine tetrahydrochloride for CD1a (a), CD3 (b), CD4 (c), CD7 (d), CD8 (e), CD20 (f), CD68 (g), and TIA-1 $(\mathbf{h})$ (original magnification $\times 200$ ). 


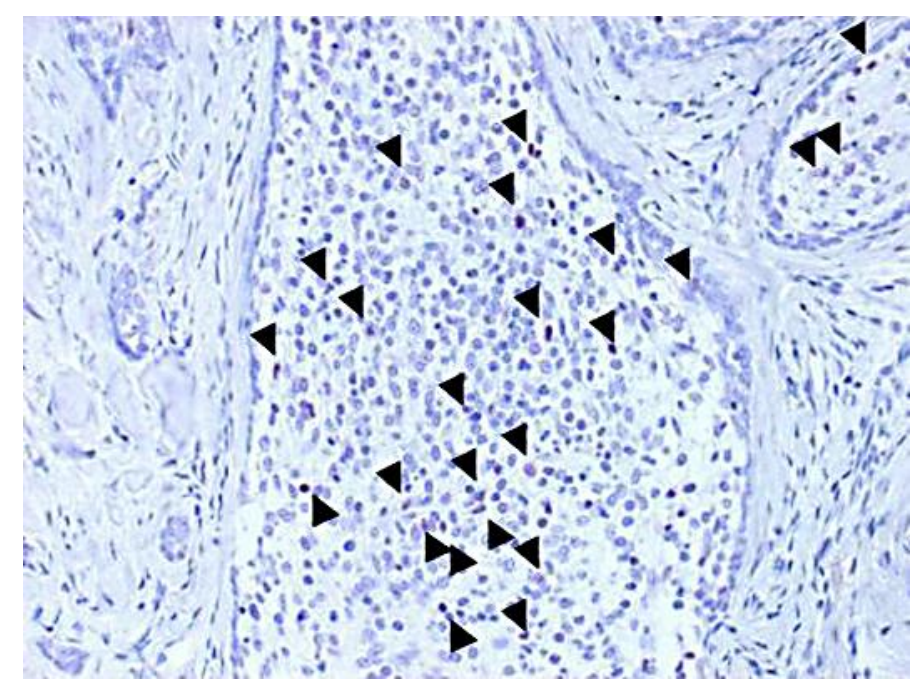

Fig. 4. Formalin-fixed paraffin-embedded tissue samples from patients with cutaneous lymphadenoma were sectioned at $4 \mu \mathrm{m}$ and deparaffinized. Deparaffinized sections were treated with autoclave heating at $121^{\circ} \mathrm{C}$ for $5 \mathrm{~min}$. After pretreatment, the sections were stained with an anti-Foxp3 Ab. The sections were developed with new fuchsin. Arrowheads indicate Foxp $3^{+}$cells (original magnification $\times 200$ ).

\section{References}

-1 Reck SJ, Xia L, Dehner LP, Reising CA: Glandular congenital lymphadenoma. Pediatr Dermatol 2007;24:547-550.

2 Santa Cruz DJ, Barr RJ, Headington JT: Cutaneous lymphadenoma. Am J Surg Pathol 1991;15:101-110.

-3 Okamura JM, Barr RJ: Cutaneous lymphoepithelial neoplasms. Adv Dermatol 1997;12:277-294.

4 Diaz-Cascajo C, Borghi S, Rey-Lopez A, Carretero-Hernandez G: Cutaneous lymphadenoma. A peculiar variant of nodular trichoblastoma. Am J Dermatopathol 1996;18:186-191.

5 Alsadhan A, Taher M, Shokravi M: Cutaneous lymphadenoma. J Am Acad Dermatol 2003;49:1115-1116.

$\checkmark 6$ Verdolini R, Stefanato CM: A pearly nodule on the upper eyelid. Clin Exp Dermatol 2010;35:561-562.

-7 Inaloz HS, Chowdhury MM, Knight AG: Cutaneous lymphadenoma. J Eur Acad Dermatol Veneoreol 2001;15:481-483.

-8 McNiff JM, Eisen RN, Glusac EJ: Immunohistochemical comparison of cutaneous lymphadenoma, trichoblastoma, and basal cell carcinoma: support for classification of lymphadenoma as a variant of trichoblastoma. J Cutan Pathol 1999;26:119-124.

99 Fujimura T, Okuyama R, Ito Y, Aiba S: Profiles of Foxp3+ regulatory T cells in eczematous dermatitis, psoriasis vulgaris and mycosis fungoides. Br J Dermatol 2008;158:1256-1263.

10 Fujimura T, Okuyama R, Ohtani T, Ito Y, Haga T, Hashimoto A, Aiba S: Perilesional treatment of metastatic melanoma with interferon-beta. Clin Exp Dermatol 2009;34:793-799. 